\title{
High Diversity Multi-Block Space-Time Code for Broadcasting Applications
}

\author{
Charbel Abdel Nour, Member, IEEE \\ Department of Electronics \\ Telecom Bretagne, Lab-STICC \\ Brest, France \\ charbel.abdelnour@telecom-bretagne.eu
}

\author{
Catherine Douillard, Member, IEEE \\ Department of Electronics \\ Telecom Bretagne, Lab-STICC \\ Brest, France \\ catherine.douillard@telecom-bretagne.eu
}

\begin{abstract}
We propose a STBC that calls for a 2 times $2 \times 2$ matrix seen as a two-block matrix. It enjoys a flexible transmission rate and a diversity order of 8 for a $2 \times 2$ MIMO system by exploiting additional time diversity. Its decoding complexity is only proportional to the power of four of the constellation size. The STBC parameters have been optimized to suit fading channels with and without erasure events. These events are typical of single frequency networks that are becoming a popular topology thanks to their low bandwidth requirements. Unlike state-of-the art quasi-orthogonal structures, the proposed multi-block STBC can be jointly optimized for shaping and coding gain.
\end{abstract}

Keywords-component; Multiple Input Multiple Output, Multiantennas, Space-Time Codes, Broadcast, SFN, Erasure, Rayleigh

\section{INTRODUCTION}

Next generation telecommunication and broadcasting systems should be designed to satisfy the need for high data rate transmissions while improving robustness to severe channel conditions. On one hand, reliable transmissions over deeply faded channels require an increase in the diversity order. On the other hand, multiple antenna systems can achieve high throughputs without requiring additional bandwidth. Therefore the introduction of Space-Time Codes (STC) for Multiple Input Multiple Output (MIMO) systems is considered to be a promising mean of fulfilling these constraints. Indeed, STC have been introduced in several communications standards like the Long Term Evolution (LTE) of the 3rd Generation Partnership Project (3GPP) [1] and the second generation of the Worldwide Interoperability for Microwave Access (WiMAX 2) [2].

Broadcast standards have not introduced full rate MIMO yet. Indeed, one major difference with respect to telecommunication systems resides in the absence of return channel carrying crucial information about transmission quality. It enables adaptive throughput via varying coding and modulation schemes. In addition, broadcast network topology can play a significant role in the definition of the constraints of the system. Indeed, Single Frequency Networks (SFN) where several transmitters simultaneously send the same signal over the same frequency channel, are increasingly used in broadcasting applications due to their efficient usage of the radio spectrum.
However, SFN entail undesirable effects on the transmission channel since their deployment leads to the introduction of deep fades. In addition to multipath in the case of a single transmitter, several echoes of the same signal are also received due to presence of several transmitters in the SFN case. The constructive or destructive addition of these echoes, also known as self-interference, results in additional deep fades. Frequently, they have a dynamic range beyond the reach of a standard receiver due to quantization error. In this case, deep fades can be modeled by erasure events causing a significant performance penalty.

A possible solution to mitigate the impact of SFN on performance can be found by using diversity techniques. Indeed, the introduction of Signal Space Diversity (SSD) for Single Input Single Output (SISO) broadcast systems has proven to improve performance for deeply faded or erased channels [3]. Therefore, MIMO for broadcasting should consider introducing STC with high diversity.

The Golden code [4] is one of the best known STBC due to its remarkable properties in terms of full rate, full diversity and having a non-vanishing determinant. However, the Maximum Likelihood (ML) decoding complexity of such a code remains relatively high especially for high order constellation schemes. Therefore, alternative STBC structures have been proposed lately [5][6][7][8][9][10] that enjoy what is known as fast decoding. They are generally based on a quasi-orthogonal structure that allows for a lower complexity decoding under a channel invariability constraint.

Based on the STBC structure of [5], we propose the introduction of SSD between modulation symbols of two STBC blocks. The resulting Multi-Block STBC (MB-STBC) is shown to enjoy full rate and a linear increase in diversity with increasing number of Blocks. By using the quasi-orthogonal structure, the proposed STBC remains fast decodable with a complexity of the same order than the golden code. In addition, it has been designed to withstand different scenarios of severe SFN channel conditions where one or more transmit antennas suffer from deep fading or erasure events during one or more symbol periods.

The paper is organized as follows. In section II, considered channel models are presented. Then, the MB-STBC structure with its corresponding low complexity decoding is described in

This work is funded by the CELTIC European project "Enabling Next GeneratIon NEtworks for broadcast Services" (ENGINES), a task force for the Digital Video Broadcasting (DVB) consortium. 
section III. Section IV explains the proposed steps for defining the MB-STBC parameters. Simulation results are presented in section V. Finally, section VI concludes the paper.

\section{CHANNEL MODELS}

Two channel models have been considered: the classical Rayleigh fading channel and a variation of this channel to which we have added additional deep fade events. Both are based on the fading channel model of [11].

\section{A. The fading channel model}

We adopt the classical frequency nonselective memoryless Rayleigh fading channel model. The received discrete time baseband complex signal $r_{j}$ on antenna $j$ can be written as:

$$
\left[\begin{array}{l}
r_{1} \\
r_{2}
\end{array}\right]=\left[\begin{array}{ll}
h_{11} & h_{12} \\
h_{21} & h_{22}
\end{array}\right]\left[\begin{array}{l}
s_{1} \\
s_{2}
\end{array}\right]+\left[\begin{array}{l}
n_{1} \\
n_{2}
\end{array}\right]
$$

where $s_{l}$ represents the complex transmitted signal on antenna $l, h_{j l}$ is a Rayleigh distributed fading coefficient with $E\left(h_{j l}^{2}\right)=1$, and $n_{j}$ is a complex white Gaussian noise with spectral density $N_{0} / 2$ in each component axis. We assume coherent detection and perfect Channel State Information (CSI) knowledge so that $h_{j l}$ and the phase of the signal are perfectly estimated and available at the receiver.

\section{B. The fading channel model with erasures}

In addition to the classical Rayleigh fading described above, additional erasure events can affect the transmitted signal. In addition, if a properly designed channel interleaver is introduced, these events can be modeled by a discrete random processes $e$ taking value 0 with a probability of $P_{e}$ and value 1 with a probability of $1-P_{e}$. The received discrete time baseband complex signal $r_{j}$ becomes:

$$
\left[\begin{array}{l}
r_{1} \\
r_{2}
\end{array}\right]=\left[\begin{array}{ll}
e_{1} h_{11} & e_{2} h_{12} \\
e_{1} h_{21} & e_{2} h_{22}
\end{array}\right]\left[\begin{array}{l}
s_{1} \\
s_{2}
\end{array}\right]+\left[\begin{array}{l}
n_{1} \\
n_{2}
\end{array}\right]
$$

The occurrence of erasure events sets a bound on the coding rate $R$. In fact, with an erasure probability of $P_{e}$ a reliable coded transmission cannot be ensured with a redundancy ratio lower than $P_{e}$, or in other words with a coding rate greater than $1-P_{e}$. This prevents the conventional system from operating at high coding rates and sets a bound on the achievable spectral efficiency.

\section{SYSTEM DESCRIPTION}

\section{A. The proposed $M B-S T B C$} form:

The proposed STBC calls for a $2 \times 4$ matrix of the following

$$
\mathbf{X}=\left[\begin{array}{llll}
s_{1}^{\prime \prime} & s_{3}^{\prime \prime} & s_{5}^{\prime \prime} & s_{7}^{\prime \prime} \\
s_{2}^{\prime \prime} & s_{4}^{\prime \prime} & s_{6}^{\prime \prime} & s_{8}^{\prime \prime}
\end{array}\right]
$$

This structure allows the transmission of 8 signals $s_{1}^{\prime \prime} \cdots s_{8}^{\prime \prime}$ through 2 antennas over 4 time slots. The first (second) row of the matrix contains the 4 signals successively sent through the first (second) transmit antenna.

We assume that the channel coefficients are constant during the two first and the two last time slots. In other words, a quasiorthogonal STBC structure spread over 4 slots. In a multicarrier transmission system, this property can be obtained by transmitting the signals of columns 1 and 2 (respectively of columns 3 and 4) of $\mathbf{X}$ over adjacent subcarriers while the signals of columns 1 (respectively 2) and 3 (respectively 4) are transmitted over distant subcarriers.

Two different channel matrices have then to be considered: $\mathrm{H}$ for the transmission of signals in columns 1 and 2 and $H$ ' for the transmission of signals in columns 3 and 4:

$$
\mathbf{H}=\left[\begin{array}{ll}
h_{11} & h_{12} \\
h_{21} & h_{22}
\end{array}\right] \text { and } \mathbf{H}^{\prime}=\left[\begin{array}{ll}
h_{11}^{\prime} & h_{12}^{\prime} \\
h_{21}^{\prime} & h_{22}^{\prime}
\end{array}\right]
$$

Let us consider 8 modulation symbols $s_{1} \ldots s_{8}$ taken from an $M$-order 2-dimensional constellation $\mathbf{C}$, where in-phase $I$ and quadrature $Q$ components are correlated. This correlation can be obtained by applying a rotation to the original constellation. The rotation angle should be chosen such that every constellation point is uniquely identifiable on each component axis separately. This is equivalent to the first step performed for SSD [3]. The representation of $s_{i}$ in the complex plane is given by $s_{i}=I_{i}+j Q_{i}, i=1 \cdots 8$. The proposed construction of $\mathrm{X}$ involves the application of two steps:

Step 1: the first step consists of defining two subsets $S_{1}^{\prime}$ and $S_{2}^{\prime}$ of modified symbols $s_{i}^{\prime}$ obtained from $I$ and $Q$ components belonging to different symbols $s_{i}$. Each subset must only contain one component of each symbol $s_{i}$ of $\mathbf{C}$. For instance:

$$
\begin{aligned}
& S_{1}^{\prime}=\left\{s_{1}^{\prime}, s_{2}^{\prime}, s_{3}^{\prime}, s_{4}^{\prime}\right\} \text { and } S_{2}^{\prime}=\left\{s_{5}^{\prime}, s_{6}^{\prime}, s_{7}^{\prime}, s_{8}^{\prime}\right\} \\
& s_{1}^{\prime}=I_{1}+j Q_{7} \quad s_{5}^{\prime}=I_{5}+j Q_{3} \\
& \text { where } \begin{array}{l}
s_{2}^{\prime}=I_{2}+j Q_{8} \\
s_{3}^{\prime}=I_{3}+j Q_{5}
\end{array} \text { and } \begin{array}{l}
s_{6}^{\prime}=I_{6}+j Q_{4} \\
s_{7}^{\prime}=I_{7}+j Q_{1}
\end{array} \\
& s_{4}^{\prime}=I_{4}+j Q_{6} \quad s_{8}^{\prime}=I_{8}+j Q_{2}
\end{aligned}
$$

Symbols $S_{j}^{\prime}$ belong to an extended constellation $\mathbf{C}^{\prime}$ of size $M^{2}$.

Step 2: the symbols $s_{1}^{\prime \prime} \cdots s_{8}^{\prime \prime}$ transmitted by $\mathrm{X}$ are defined as

$$
\begin{array}{ll}
s_{1}^{\prime \prime}=a s_{1}^{\prime}+b s_{2}^{\prime} & s_{5}^{\prime \prime}=a s_{5}^{\prime}+b s_{6}^{\prime} \\
s_{2}^{\prime \prime}=a s_{3}^{\prime}+b s_{4}^{\prime} & s_{6}^{\prime \prime}=a s_{7}^{\prime}+b s_{8}^{\prime} \\
s_{3}^{\prime \prime}=-c s_{3}^{\prime *}-d s_{4}^{\prime *} & \text { and } \begin{array}{l}
s_{7}^{\prime \prime}=-c s_{7}^{\prime *}-d s_{8}^{\prime *} \\
s_{4}^{\prime \prime}=c s_{1}^{\prime *}+d s_{2}^{\prime *}
\end{array} s_{8}^{\prime \prime}=c s_{5}^{\prime *}+d s_{6}^{\prime *}
\end{array}
$$


where $s^{*}$ represents the complex conjugate of $S$.

$a, b, c$ and $d$ are complex-valued parameters of the STBC. Signals $S^{\prime \prime}$ belong to the STBC constellation signal set $\mathbf{C}^{\prime \prime}$ different from $\mathbf{C}^{\prime}$.

\section{B. Decoding the $M B-S T B C$ code}

The proposed MB-STBC code enjoys a structure that enables a simplified detection. Indeed, inspired by the decoding process in [5], the decoding complexity can be greatly simplified without the need for a sphere decoder [12]. If we denote by $r_{k}^{j}$ the signal received by the $j^{\text {th }}$ reception antenna, $j=1,2$, during time slot $k$, where $k=1 \ldots 4$.

The four signals successively received by antenna 1 can be written as:

$$
\begin{aligned}
r_{1}^{1}= & h_{11}\left[a\left(I_{1}+j Q_{7}\right)+b\left(I_{2}+j Q_{8}\right)\right] \\
& +h_{12}\left[a\left(I_{3}+j Q_{5}\right)+b\left(I_{4}+j Q_{6}\right)\right]+n_{1}^{1} \\
r_{2}^{1}= & h_{11}\left[-c\left(I_{3}-j Q_{5}\right)-d\left(I_{4}-j Q_{6}\right)\right] \\
& +h_{12}\left[c\left(I_{1}-j Q_{7}\right)+d\left(I_{2}-j Q_{8}\right)\right]+n_{2}^{1} \\
r_{3}^{1}= & h_{11}^{\prime}\left[a\left(I_{5}+j Q_{3}\right)+b\left(I_{6}+j Q_{4}\right)\right] \\
& +h_{12}^{\prime}\left[a\left(I_{7}+j Q_{1}\right)+b\left(I_{8}+j Q_{2}\right)\right]+n_{3}^{1} \\
r_{4}^{1}= & h_{11}^{\prime}\left[-c\left(I_{7}-j Q_{1}\right)-d\left(I_{8}-j Q_{2}\right)\right] \\
& +h_{12}^{\prime}\left[c\left(I_{5}-j Q_{3}\right)+d\left(I_{6}-j Q_{4}\right)\right]+n_{4}^{1}
\end{aligned}
$$

Simplified decoding is possible under the condition that the $I$ and $Q$ components of any $s_{i}$ constellation symbol are mapped to two different $s$ 'symbols who are multiplied by the same STBC parameter $a, b, c$ or $d$. This constraint is respected in the structure of the STBC matrix $\mathbf{X}$. Therefore, by re-arranging equations (5) to (8) we obtain $y_{k}^{j}$ terms:

$$
\begin{aligned}
y_{1}^{1} & =r_{1}^{1}-b\left[h_{11}\left(I_{2}+j Q_{8}\right)+h_{12}\left(I_{4}+j Q_{6}\right)\right] \\
& =a\left[h_{11}\left(I_{1}+j Q_{7}\right)+h_{12}\left(I_{3}+j Q_{5}\right)\right]+n_{1}^{1} \\
y_{2}^{1} & =r_{2}^{1}-d\left[h_{12}\left(I_{2}-j Q_{8}\right)-h_{11}\left(I_{4}-j Q_{6}\right)\right] \\
& =c\left[h_{12}\left(I_{1}-j Q_{7}\right)-h_{11}\left(I_{3}-j Q_{5}\right)\right]+n_{2}^{1} \\
y_{3}^{1} & =r_{3}^{1}-b\left[h_{11}^{\prime}\left(I_{6}+j Q_{4}\right)+h_{12}^{\prime}\left(I_{8}+j Q_{2}\right)\right] \\
& =a\left[h_{11}^{\prime}\left(I_{5}+j Q_{3}\right)+h_{12}^{\prime}\left(I_{7}+j Q_{1}\right)\right]+n_{3}^{1} \\
y_{4}^{1} & =r_{4}^{1}-d\left[h_{12}^{\prime}\left(I_{6}-j Q_{4}\right)-h_{11}^{\prime}\left(I_{8}-j Q_{2}\right)\right] \\
& =c\left[h_{12}^{\prime}\left(I_{5}-j Q_{3}\right)-h_{11}^{\prime}\left(I_{7}-j Q_{1}\right)\right]+n_{4}^{1}
\end{aligned}
$$

In equations (9) to (12), the first line terms are only dependent on $I$ and $Q$ components of even symbols $s$. Viceversa, second line terms depend solely on odd symbols. Therefore, applying a detection conditioned by the knowledge of even terms is possible. In other words, for a loop on all possible values for $S_{2}=I_{2}+j Q_{2}, S_{4}=I_{4}+j Q_{4}$, $S_{6}=I_{6}+j Q_{6}$ and $S_{8}=I_{8}+j Q_{8}$ (for a total of $M^{4}$ terms where
$M$ represents the order of the constellation $s$ ) intermediate $\mathrm{Z}_{\mathrm{k}}$ terms can be computed as follows:

$$
\begin{aligned}
& Z_{1}=\frac{h_{11}^{*} y_{1}^{1}+h_{21}^{*} y_{1}^{2}}{a}+\frac{h_{12} y_{2}^{1^{*}}+h_{22} y_{2}^{2^{*}}}{c^{*}} \\
& Z_{2}=\frac{h_{12}^{*} y_{1}^{1}+h_{22}^{*} y_{1}^{2}}{a}-\frac{h_{11} y_{2}^{1^{*}}+h_{21} y_{2}^{2^{*}}}{c^{*}} \\
& Z_{3}=\frac{h_{11}^{\prime *} y_{3}^{1}+h_{21}^{\prime *} y_{3}^{2}}{a}+\frac{h_{12}^{\prime} y_{4}^{1^{*}}+h_{22}^{\prime} y_{4}^{2^{*}}}{c^{*}} \\
& Z_{4}=\frac{h_{12}^{\prime *} y_{3}^{1}+h_{22}^{\prime *} y_{3}^{2}}{a}-\frac{h_{11}^{\prime} y_{4}^{1^{*}}+h_{21}^{\prime} y_{4}^{2^{*}}}{c^{*}}
\end{aligned}
$$

By properly combining $Z_{k}$ terms, we obtain:

$$
\begin{aligned}
& \operatorname{Re}\left\{Z_{1}\right\}+j \operatorname{Im}\left\{Z_{4}\right\}=\left(\left|h_{11}\right|^{2}+\left|h_{12}\right|^{2}+\left|h_{21}\right|^{2}+\left|h_{22}\right|^{2}\right) I_{1} \\
& +j\left(\left|h_{11}^{\prime}\right|^{2}+\left|h_{12}^{\prime}\right|^{2}+\left|h_{21}^{\prime}\right|^{2}+\left|h_{22}^{\prime}\right|^{2}\right) Q_{1}+\operatorname{Re}\left\{N_{1}\right\}+j \operatorname{Im}\left\{N_{4}\right\} \\
& \operatorname{Re}\left\{Z_{2}\right\}+j \operatorname{Im}\left\{Z_{3}\right\}=\left(\left|h_{11}\right|^{2}+\left|h_{12}\right|^{2}+\left|h_{21}\right|^{2}+\left|h_{22}\right|^{2}\right) I_{3} \\
& +j\left(\left|h_{11}^{\prime}\right|^{2}+\left|h_{12}^{\prime}\right|^{2}+\left|h_{21}^{\prime}\right|^{2}+\left|h_{22}^{\prime}\right|^{2}\right) Q_{3}+\operatorname{Re}\left\{N_{2}\right\}+j \operatorname{Im}\left\{N_{3}\right\} \\
& \operatorname{Re}\left\{Z_{3}\right\}+j \operatorname{Im}\left\{Z_{2}\right\}=\left(\left|h_{11}^{\prime}\right|^{2}+\left|h_{12}^{\prime}\right|^{2}+\left|h_{21}^{\prime}\right|^{2}+\left|h_{22}^{\prime}\right|^{2}\right) I_{5} \\
& +j\left(\left|h_{11}\right|^{2}+\left|h_{12}\right|^{2}+\left|h_{21}\right|^{2}+\left|h_{22}\right|^{2}\right) Q_{5}+\operatorname{Re}\left\{N_{3}\right\}+j \operatorname{Im}\left\{N_{2}\right\} \\
& \operatorname{Re}\left\{Z_{4}\right\}+j \operatorname{Im}\left\{Z_{1}\right\}=\left(\left|h_{11}^{\prime}\right|^{2}+\left|h_{12}^{\prime}\right|^{2}+\left|h_{21}^{\prime}\right|^{2}+\left|h_{22}^{\prime}\right|^{2}\right) I_{7} \\
& +j\left(\left|h_{11}\right|^{2}+\left|h_{12}\right|^{2}+\left|h_{21}\right|^{2}+\left|h_{22}\right|^{2}\right) Q_{7}+\operatorname{Re}\left\{N_{4}\right\}+j \operatorname{Im}\left\{N_{1}\right\}
\end{aligned}
$$

With the noise terms $N_{k}$ being:

$$
\begin{aligned}
& N_{1}=\frac{h_{11}^{*} n_{1}^{1}+h_{21}^{*} n_{1}^{2}}{a}+\frac{h_{12} n_{2}^{1^{*}}+h_{22} n_{2}^{2^{*}}}{c^{*}} \\
& N_{2}=\frac{h_{12}^{*} n_{1}^{1}+h_{22}^{*} n_{1}^{2}}{a}-\frac{h_{11} n_{2}^{1^{*}}+h_{21} n_{2}^{2^{*}}}{c^{*}} \\
& N_{3}=\frac{h_{11}^{\prime *} n_{3}^{1}+h_{21}^{\prime *} n_{3}^{2}}{a}+\frac{h_{12}^{\prime} n_{4}^{1^{*}}+h_{22}^{\prime} n_{4}^{2^{*}}}{c^{*}} \\
& N_{4}=\frac{h_{12}^{\prime *} n_{3}^{1}+h_{22}^{\prime *} n_{3}^{2}}{a}-\frac{h_{11}^{\prime} n_{4}^{1^{*}}+h_{21}^{\prime} n_{4}^{2^{*}}}{c^{*}}
\end{aligned}
$$

Equations (17) to (20) show that the combinations of $\mathrm{Z}_{\mathrm{k}}$ dependent terms are each a function of only one $s_{j}=I_{j}+j Q_{j}$ symbol. Therefore a simple linear detection can be performed separately on all symbols in the same loop since every $I_{i}$ and $Q_{i}$ couple is unique. In addition, the diversity of 8 is clearly observed since the $I$ and $Q$ components of every symbol depend on 4 different channel coefficients. Therefore, since SSD is applied, every complex $s_{i}$ signal enjoys an overall diversity of 8 .

The detection of odd symbols on the second antenna is similar to the first antenna. For the joint detection of even symbols, the following distance should be minimized: 


$$
\begin{aligned}
D\left(s_{2}, s_{4}, s_{6}, s_{8}\right)= & \left|y_{1}^{1}-a\left[h_{11}\left(I_{1}+j Q_{7}\right)+h_{12}\left(I_{3}+j Q_{5}\right)\right]\right|^{2} \\
& +\left|y_{2}^{1}-c\left[h_{12}\left(I_{1}-j Q_{7}\right)-h_{11}\left(I_{3}-j Q_{5}\right)\right]\right|^{2} \\
& +\left|y_{3}^{1}-a\left[h_{11}^{\prime}\left(I_{5}+j Q_{3}\right)+h_{12}^{\prime}\left(I_{7}+j Q_{1}\right)\right]\right|^{2} \\
& +\left|y_{4}^{1}-c\left[h_{12}^{\prime}\left(I_{5}-j Q_{3}\right)-h_{11}^{\prime}\left(I_{7}-j Q_{1}\right)\right]\right|^{2} \\
& +\left|y_{1}^{2}-a\left[h_{21}\left(I_{1}+j Q_{7}\right)+h_{22}\left(I_{3}+j Q_{5}\right)\right]\right|^{2} \\
& +\left|y_{2}^{2}-c\left[h_{22}\left(I_{1}-j Q_{7}\right)-h_{21}\left(I_{3}-j Q_{5}\right)\right]\right|^{2} \\
& +\left|y_{3}^{2}-a\left[h_{21}^{\prime}\left(I_{5}+j Q_{3}\right)+h_{22}^{\prime}\left(I_{7}+j Q_{1}\right)\right]\right|^{2} \\
& +\left|y_{4}^{2}-c\left[h_{22}^{\prime}\left(I_{5}-j Q_{3}\right)-h_{21}^{\prime}\left(I_{7}-j Q_{1}\right)\right]\right|^{2}
\end{aligned}
$$

The distance $D\left(s_{2}, s_{4}, s_{6}, s_{8}\right)$ of equation (21) can be directly computed from $y_{k}^{j}$ terms (which depend on $s_{2}, s_{4}, s_{6}$ and $s_{8}$ ) of equations (9) to (12) and by replacing the $I$ and $Q$ components of odd constellation symbol terms by their detected values from equations (17) to (20). Since $D\left(s_{2}, s_{4}, s_{6}, s_{8}\right)$ should be computed for all possible combinations of even constellation symbols, the total number of computed terms is in the order of $M^{4}$.

Note that the simplified detection does not depend on the choice of the STBC parameters $a, b, c$ and $d$. These should be chosen depending on the rank, determinant, and shaping considerations.

\section{DEFINITION OF MB-STBC PARAMETERS}

\section{A. Conventional STBC design criteria}

The minimization of the union bound for the Pairwise Error Probability (PEP) leads to the well-known rank-determinant [13]:

1) Rank Criterion: To achieve the maximum diversity, the rank $R_{a}$ of the codeword difference matrix $\Delta=\mathbf{X}-\hat{\mathbf{X}}$ must be maximized for all possible transmitted codeword pairs $(\mathbf{X}, \hat{\mathbf{X}})$. When $R_{a}=N_{t}$ ( $N_{t}$ being the number of transmit antennas), the $\mathrm{STBC}$ is said to reach full diversity.

2) Determinant Criterion: The STBC minimum determinant $\delta$ is defined as:

$$
\delta=\min _{\mathbf{X} \neq \hat{\mathbf{X}}} \operatorname{det}\left[(\mathbf{X}-\hat{\mathbf{X}})(\mathbf{X}-\hat{\mathbf{X}})^{H}\right]
$$

Where $H$ denotes the Hermitian transpose. For high SNRs, in order to obtain the best performance, $\delta$ should be maximized. Indeed, the dominant parameter is the diversity gain which defines the slope of error rate curves. Therefore, it is important to ensure the full diversity of the STBC and then maximize its coding gain $\delta^{1 / N_{t}}$.

The search for the minimum determinant is performed over an ensemble that spans all possible values of $\mathbf{X}$ and $\mathbf{X}^{\prime}$. For the proposed MB-STBC, this ensemble includes $M^{16}$ different values. Taking into account that the STBC is linear and that the difference between two STBC codewords is a codeword, a simplified determinant criterion can be computed as follows:

$$
\delta^{\prime}=\min _{\mathbf{X}} \operatorname{det}\left[\mathbf{X} \mathbf{X}^{H}\right]
$$

The search over $\delta^{\prime}$ reduces the search ensemble to $M^{8}$ values.

\section{B. STBC design criteria for channels with erasures}

Designing STBC codes that can withstand erasure events follows the same procedure as the conventional criteria followed by additional steps based on the computation of the simplified determinant criteria where the erased position(s) are replaced by null value(s). The resulting $\mathbf{X}$ matrix parameters should be chosen such that the simplified determinant remains as high as possible. Four cases have been considered in our study:

Case 1: A classical Rayleigh fading channel as defined in section II-A. The conventional STBC design criteria apply.

Case 2: A classical Rayleigh fading channel with one erased transmit antenna during two symbol periods. The channel follows section II-B for the erased antenna. The erasure event spans two symbol periods due to the quasi-orthogonal structure of the STBC.

Case 3: A classical Rayleigh fading channel with one erased transmit antenna during the four symbol periods (all of the time).

Case 4: A classical Rayleigh fading channel with both transmit antennas erased during two symbol periods.

\section{Choice of MB-STBC parameters}

The choice of STBC parameters follows several constraints:

- Transmit power of each antenna is normalized.

- Rotation angle for SSD.

- $|a|=|b|=|c|=|d|=\frac{1}{\sqrt{2}}$ in order to guarantee identical transmit average energy per antenna .

- Having to divide by $a$ and $c^{*}$ during the simplified detection process (equ (13) to equ (16)), we have chosen these two parameters to be real valued and therefore $a=c=1 / \sqrt{2}$.

- $d=e^{-j \frac{\pi}{2}} b$, or angle $(d)=\operatorname{angle}(b)-\pi / 2$. Therefore, the search space is limited to finding the angle of $b$ : angle $(b)=\Phi$ satisfying the design criteria

On one side, parameters $a$ et $c$ being real numbers should not be a limiting factor for the search space. Indeed, the chosen angle $(b)=\Phi$ values satisfying the constraints are still valid when $a$ and $c$ are complex numbers. Indeed, the only requirement is that $a, b, c, d$ parameters respect the following constraint: 
$\operatorname{angle}(a)=\Phi_{\mathrm{a}}, \quad \operatorname{angle}(c)=-\Phi_{\mathrm{a}}, \quad \operatorname{angle}(b)=\Phi+\Phi_{\mathrm{a}} \quad$ and angle $(d)=\Phi-\Phi_{\mathrm{a}}-\pi / 2$, whatever the value taken by $\Phi_{\mathrm{a}}$.

On the other side, search for the angle $(b)=\Phi$ can be limited to the interval $[0, \pi]$ due to the symmetry of the $C^{\prime \prime}$ constellation with respect to axes origin. Consequently, angles $\alpha$ and $\alpha+\pi$ result into equivalent STBC codes with respect to the design criteria.

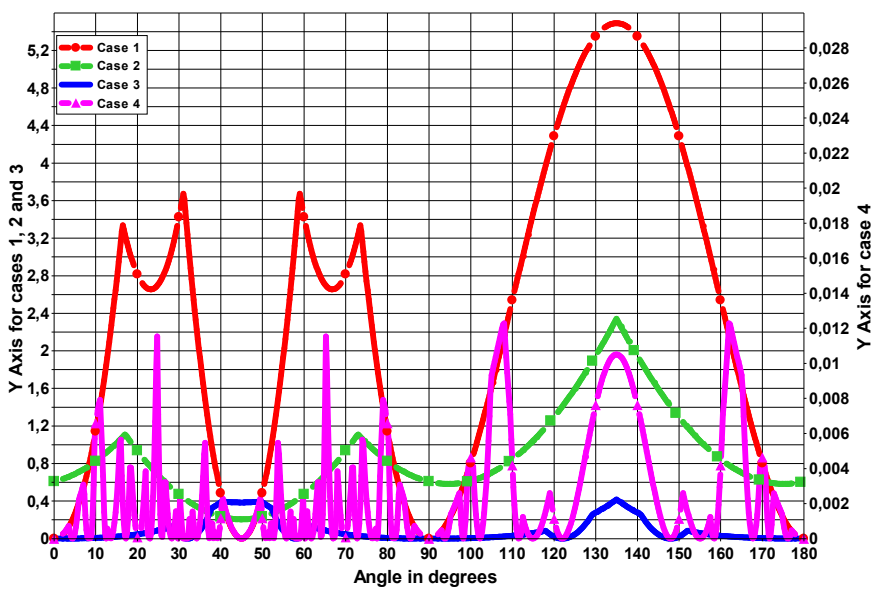

Figure 1. Simplified minimum determinant criteria as a function of angle $(b)=\Phi$ for the 4 considered cases of section IV-B

The minimum determinant as a function of the angle of parameter $b$ for the 4 different cases of section IV-B is shown in fig. 1. For cases 1,2 and $3, \Phi=135^{\circ}$ represents the value that maximizes the simplified determinant. For this same angle, MB-STBC achieves one of the highest determinant values for case 4. Therefore, angle $(b)=\Phi=135^{\circ}$ can be considered as a compromise that enables good performance for the proposed MB-STBC structure under considered scenarios.

In addition, MB-STBC with $\Phi=135^{\circ}$ enjoys a high shaping gain. Indeed, the introduction of SSD increases the number of possible constellation points to be transmitted on every antenna. Parameters should be chosen such that this shaping gain is as high as possible since it plays a role on performance for low Eb/N0 values. Unlike classical $2 \times 2$ quasiorthogonal structures where the shaping gain and the coding gain are in general competing and not complementing, the MBSTBC with $\Phi=135^{\circ}$ shows the best coding and shaping gain since the resulting code exhibits the best error rate performance for low and high $\mathrm{Eb} / \mathrm{N} 0$ regions among all $\Phi$ values leading to non-zero rank.

\section{SimUlation RESUlTS}

Simulations have been carried out over a Rayleigh fading channel with and without erasures. When erasures were simulated, studied cases in section IV-B were considered. The erasure events are then applied on an antenna all of the time. This is equivalent to having $P_{\mathrm{e}}=1$ in section II-B. These two simulated cases represent extreme cases since a realistic scenario should lie between $P_{\mathrm{e}}=0$ (Rayleigh channel) and $P_{\mathrm{e}}=$ 1 (Rayleigh channel with erased antennas all of the time).

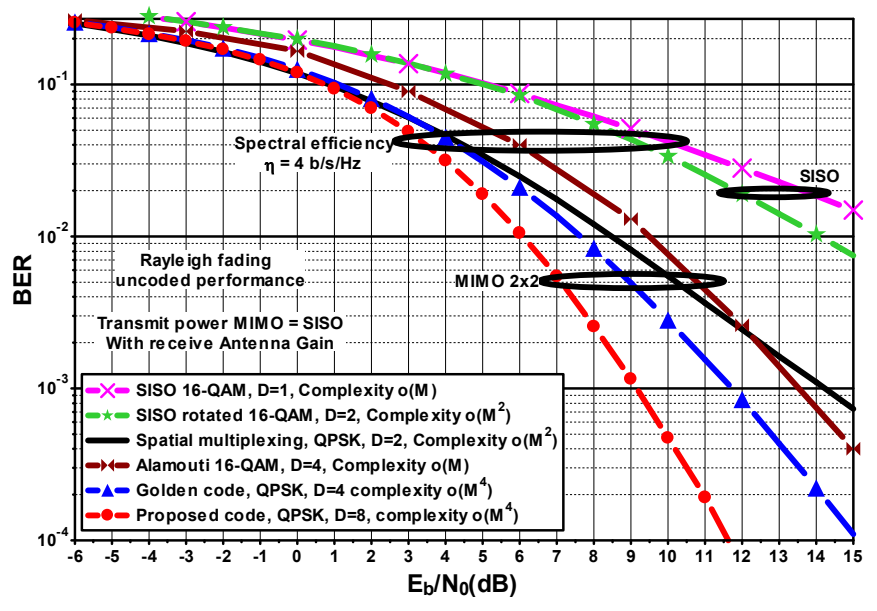

Figure 2. BER comparison between proposed MB-STBC, Alamouti 2x2, Golden code, spatial multiplexing, SISO and SISO with SSD over a Rayleigh fading channel for 4 bits/channel use.

A comparison in terms of Bit Error rate (BER) between the proposed MB-STBC, the Alamouti $2 \mathrm{X} 2$ code, the Golden code, spatial multiplexing, SISO and SISO with SSD are presented in fig. 2 over a Rayleigh fading channel for a spectral efficiency of 4 bits/channel use. It can be observed that the proposed MB$\mathrm{STBC}$ outperforms all codes for high $\mathrm{Eb} / \mathrm{N} 0$ values since it achieves a diversity order of 8 .

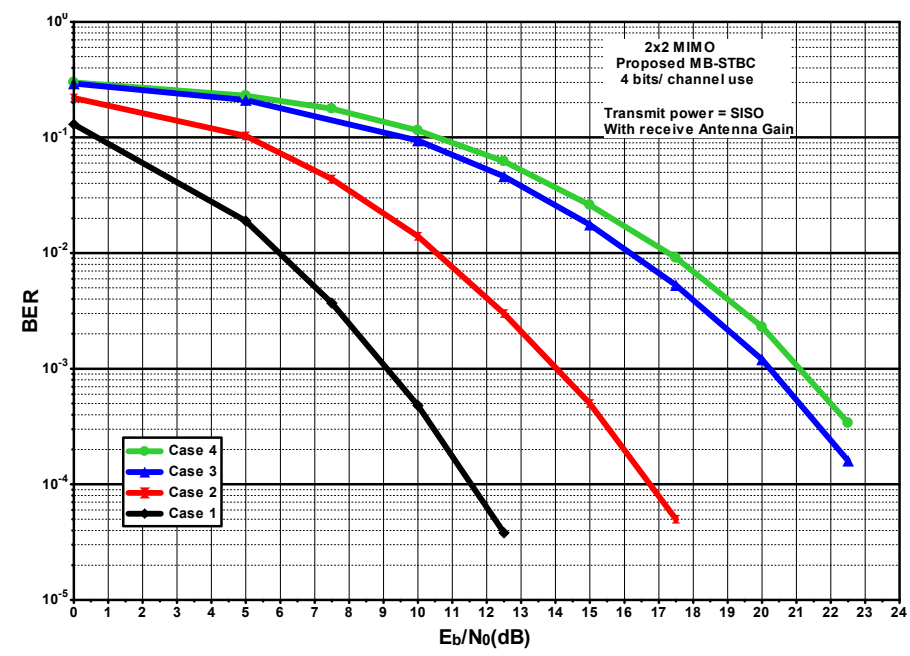

Figure 3. BER performance of the proposed MB-STBC, for cases 1, 2, 3 and 4 of section IV-B and 4 bits/channel use.

Fig. 4 shows BER performance of the MB-STBC for the 4 different channel cases. Despite the extreme nature of considered scenarios, the proposed code is still able to detect all transmitted constellation symbols. It achieves a diversity order of 4 for cases 3 and 4 where half of the transmitted signal power is not received.

When concatenated with a powerful outer Forward Error Correcting (FEC) code that also exploits time and spatial diversity, the gain of the proposed STBC will depend on the actual coding rate and the amount of erasure events. Indeed, as mentioned in section II.B, the amount of erasure events or $P_{e}$ sets a bound on the FEC coding rate. If the chosen rate is larger 
than this bound, the FEC decoder will not achieve iterative convergence unless concatenated with the proposed STBC.

\section{CONCLUSION}

We have proposed a new multi-block space-time block code that follows a quasi-orthogonal structure and achieves a diversity order of 8 for a $2 \times 2$ MIMO system by exploiting additional time/frequency diversity. Its decoding complexity remains reasonable thanks to its structure that allows for a simplified detection. Its parameters have been optimized under several scenarios where the transmit antennas can suffer from erasure events. These scenarios are particularly interesting for a transmission over single frequency networks. The resulting code compares favorably to existing STBC codes and exhibits high robustness to erasure events.

\section{REFERENCES}

[1] LTE; Evolved Universal Terrestrial Radio Access (E-UTRA); Physical layer procedures, 3GPP TS 36.213, Version 10.3.0, Release 10, Oct. 201

[2] IEEE $302.16 \mathrm{~m}$, IEEE Standard for local and metropolitan area networks - Part 16: Air Interface for Broadband Wireless Access Systems, Amendment 3: Advanced Air Interface, May 2011.

[3] C. Abdel Nour and C. Douillard, "Improving BICM Performance of QAM constellations for broadcasting applications," Int. Symp. on Turbo Codes and Iterative Techniquee, Lausanne, Switzerland, Sept. 2008.
[4] J. C. Belfiore, G. Rekaya, and E. Viterbo, "The Golden code: a $2 \times 2$ fullrate space-time code with non vanishing determinants", IEEE Trans. Inform. Theory, vol. 51, n 4, pp. 1432-1436, April 2005.

[5] S. Sezginer and H. Sari, "Full-rate full-diversity $2 \times 2$ space-time codes of reduced decoder complexity," IEEE Commun. Lett., vol. 11, no. 12, pp. 973-975, Dec. 2007.

[6] J. Paredes, A.B. Gershman, and M. G. Alkhanari, “A 2x2 space--time code with non-vanishing determinants and fast maximum likelihood decoding," in Proc IEEE Int. Conf. on Acoustics, Speech, and Signal Processing (ICASSP2007), Honolulu, Hawaii, USA, pp. 877-880, April 2007.

[7] M. Samuel and M. P. Fitz, "'Reducing the detection complexity by using $2 \times 2$ multi-strata space--time codes," in Proc IEEE Int. Symp. Inform Theory (ISIT 2007), pp. 1946-1950, Nice, France, June 2007.

[8] E. Biglieri, Y. Hong and E. Viterbo, "On fast-decodable space-time block codes,"IEEE Trans. On Information Theory, pp. 524-530, vol. 55, n. 2, Feb. 2009.

[9] C. Hollanti, J. Lahtonen, K. Ranto, R. Vehkalahti, and E. Viterbo, “On the Algebraic Structure of the Silver Code," in IEEE Information Theory Workshop, Porto, Portugal, May 2008.

[10] O. Tirkkonen and A. Hottinen, "Square-matrix embeddable space-time block codes for complex signal constellations," in IEEE Trans. Inform. Theory, vol. 48, no. 2, , pp. 384-395, February 2002.

[11] W. C. Jakes, Microwave Mobile Communications, Wiley-Interscience, May 1994.

[12] B. M. Hochwald and S. ten Brink, "Achieving near-capacity on a multiple-antenna channel," IEEE Trans. Communications, vol. 51, pp. 389-399, Mar. 2003.

[13] V. Tarokh, N. Seshadri, and A. R. Calderbank, "Space-time codes for high data rate wireless communications: Performance criterion and code construction," IEEE Trans. Inf. Theory, vol. 44, no. 2, pp. 744-765, Mar. 1998. 